AIAA 2002-1525

\title{
BUCKLING DESIGN STUDIES OF INVERTED, OBLATE BULKHEADS FOR A PROPELLANT TANK
}

\author{
Stanley S. Smeltzer III \\ NASA Langley Research Center \\ Hampton, VA 23681-2199 \\ and \\ Lynn M. Bowman ${ }^{\dagger}$ \\ Lockheed Martin Engineering and Sciences Corporation \\ Hampton, VA 23681-2199
}

\begin{abstract}
$\underline{\text { Abstract }}$
An investigation of the deformation and buckling characteristics of a composite, oblate bulkhead that has an inverted geometry and is subjected to pressure-only loading is presented for three bulkhead geometries and thicknesses. The effects of a stiffening support ring at the bulkhead to cylinder interface are also evaluated. Buckling analyses conducted using the axisymmetric shell code BOSOR4 are discussed for several bulkhead configurations. These results are analytically verified using results from the Structural Analysis of General Shells (STAGS) code for a selected bulkhead configuration.

The buckling characterization of an inverted, oblate bulkhead requires careful attention as small changes in bulkhead parameters can have a significant effect on the critical buckling load. Comparison of BOSOR4 and STAGS results provided a very good correlation between the two analysis methods. In addition, the analysis code BOSOR4 was found to be an efficient sizing tool that is useful during the preliminary design stage of a practical shell structure. Together, these two aspects should give the design engineer confidence in sizing these stability critical structures. Additional characterization is warranted, especially for a composite tank structure, since only one bulkhead configuration was examined closely.
\end{abstract}

\section{Primary Symbols}

a ellipsoidal major axis (in.)

b ellipsoidal minor axis (in.)
E Young's modulus of elasticity(psi.)

$\mathrm{G} \quad$ shear modulus (psi.)

$\hat{n}$ normal vector

$P \quad$ internal tank pressure (psi.)

$r \quad$ tank radius (in.)

S arc length

z tank longitudinal axis

Introduction

The past decade has provided numerous studies that have identified various reusable launch vehicle (RLV) configurations ${ }^{1-3}$ as well as strategies intended to optimize their performance. ${ }^{4-6}$ An important component in each one of these vehicle optimization strategies is the willingness to trade increased structural component weight for an overall reduction in vehicle weight or vehicle performance. All of these endeavors have been conducted with one goal in mind: to develop a RLV capable of providing cost-effective $(\$ 1000 / \mathrm{lbm})$ access to low-earth-orbit by reducing launch and operations costs. One of the important areas related to the development of a future reusable launch vehicle is the capability for accurately predicting optimum vehicle weight based on a variety of component configurations; such as tanks, intertanks, or thrust structures.

The current strategy for the RLV program appears to be centered about a vertical-takeoff, horizontal landing, single-stage-to-orbit, winged-body derivative that uses rocket propulsion. This being the case, conventional launch vehicle loading will be relied upon for designing major structural components, such as tanks. Improvements in structural efficiency for these

\footnotetext{
"Aerospace Engineer, Mechanics and Durability Branch. Member, AIAA and ASME.

Aeronautical Engineer.

Copyright $(2001$ by the American Institute of Aeronautics and Astronautics, Inc. No copyright is asserted in the United States under Title 17 , U.S. Code. The U.S. Government has a royalty-free license to exercise all rights under the copyright claimed herein for Governmental Purposes. All other rights are reserved by the copyright owner.
} 
components are typically obtained through geometric changes to structure or the use of advanced materials. Geometric changes are used to derive an optimum balance between increased component weight and improved system performance, while material substitutions offer improved strength and elastic modulus, lower density, and more desirable coefficients of thermal expansion and conductivity.

A key component in the design of a propellant tank is the response characterization of the bulkheads. Studies of previous RLV configurations have utilized conformal tanks and oblate bulkheads ${ }^{7,8}$ to reduce overall vehicle weight and to improve system performance. In the work by Sisk and $\mathrm{Wu}$, the shapes of the bulkheads ranged from hemispherical to hemiellipsoidal in geometry and were attached to the cylindrical portion of the tank in a manner that is conventional for propellant tanks of launch vehicles. That is, the center of curvature for the bulkheads was located inside the tank volume. A different bulkhead geometry that is more common to the pressure vessel industry reverses the direction of the radius of curvature so that the bulkhead has an inverted shape, like that on the bottom of an aluminum beverage can. This type of bulkhead geometry, hereafter referred to as inverted, is shown in Figure 1 along with conventional bulkhead geometry.

An inverted bulkhead geometry is important for the vehicle optimization strategy since an inverted bulkhead provides additional features to the propellant design that are not available from a conventional bulkhead. A few advantages offered by an inverted bulkhead, that provide an opportunity for reducing weight during the vehicle optimization, are 1) a capability for nesting adjacent tanks, 2) additional volume for hardware between tanks and intertanks, and 3) the capability for improving the fabrication and performance of interface joints. For example, an inverted bulkhead geometry offers potential improvements to the performance of adhesively bonded joints at the bulkhead to cylinder interface (i.e., Y-joint) because the internal pressure loading of the tank creates a deformed bulkhead shape that results in out-of-plane compression at the joint. Thus, peel stresses that can lead to premature joint failure are suppressed by the out-of-plane compressive response from the bulkhead, which leads to improved joint performance. However, to capitalize on these advantages a thorough knowledge of the response of an inverted bulkhead is paramount. Although a significant portion of literature exists from the industrial pressure vessel and piping community related to the buckling behavior of externally pressurized spherical, torispherical, hemispherical, and hemiellipsoidal bulkheads, there is no available literature that addresses the design, analysis, or use of oblate, hemiellipsoidal bulkheads for launch vehicle applications. Additionally, all of the previous research has focused on bulkheads of various shapes that are made of a metallic material. Thus, a need exists to characterize the behavior of oblate, hemiellipsoidal bulkheads that are inverted, made of composite materials, and have representative launch vehicle geometry and loading conditions.

The existing literature related to the behavior of inverted bulkheads is primarily concerned with the buckling and collapse of these structures in a mode referred to by Corona" as "dome reversal." All the early buckling investigations were performed on bulkhead configurations that had a spherical radius of curvature for the shell segments involved. A classical investigation by Budiansky ${ }^{10}$ characterized and solved the axisymmetric collapse of clamped spherical shells, while Hutchinson ${ }^{11}$ identified the unstable and imperfection sensitive behavior of post-buckled spherical shells. Several experimental studies ${ }^{12-15}$ investigated the buckling behavior of spherical, hemispherical, and torispherical bulkheads subjected to hydrostatic pressure. Galletly ${ }^{16}$ et al., Blachut ${ }^{17-19}$ et al., and $\mathrm{Lu}^{20}$ have more recently performed numerical and experimental studies to determine the effects of imperfections and local features on the buckling of spherical shells. One other notable area of research related to the buckling of inverted bulkheads is the work on dynamic buckling of oblate and prolate domes by $\operatorname{Ross}^{21}$, et al.

The investigations by Corona and Ross et al. are the only ones that characterized the buckling behavior of oblate, hemiellipsoidal bulkheads. Corona performed an experimental study of a low profile, hemiellipsoidal bulkhead configuration that included a nonlinear axisymmetric analysis. Although a buckled mode shape for the bulkhead was not given, the experimental results determined that the failure mode for the bulkhead occurred as a rapid collapse followed by a partial reversal of the dome upon reaching the limit point. In the work by Ross et al., the primary objective of the investigation was to determine the buckling pressures and mode shapes for an array of prolate and oblate bulkheads due to pressure and a vibratory excitation of the shell wall. However, a brief description of the static buckling response and critical pressure for each of the bulkhead shapes was given.

The objective of the present paper is to state results from an analytical investigation to determine the deformation and buckling characteristics of a composite, oblate bulkhead that has an inverted geometry and is subjected to pressure-only loading. In the remainder of the paper, a description of the overall 
tank configuration and loading, a summary of the different types of analyses used in the investigation, and a discussion of the results are given.

\section{Bulkhead Configuration and Loading}

The configuration of the bulkhead that was considered in the present study corresponds to a scaled propellant tank for a launch vehicle. Specifically, the tank geometry and pressure were scaled to represent a typical full-sized cryogenic tank for a reusable launch vehicle. The tank and inverted bulkhead geometry are depicted in Figure 1 along with the applied loading of only internal pressure. The tank internal pressure $(P)$ was 216 psi. while the right circular cylindrical portion of the tank as well as the inverted bulkhead had a radial diameter (2a) of 36 inches. The shape of the bulkhead varied from a hemispherical to hemiellipsoidal geometry; in addition, a detailed account of the different bulkhead shapes used in this investigation is given in the section: Description of Bulkhead Modeling and Analyses. The material properties for the tank were representative of a non-autoclave cured, carbon fiber reinforced plastic (CFRP) composite material system with a quasi-isotropic laminate configuration. This laminate configuration was used to represent the shell wall for both the cylinder and bulkhead structures for each of the analyses and did not include the effects of material or geometrical imperfections. As discussed by Hilburger and Starnes $^{22}$, the effects of initial geometric imperfections may have a significant influence on the buckling response of shells; however, there was no attempt made in the present investigation to quantify the effects due to geometric imperfections. The in-plane material properties for the bulkhead and cylinder used in this investigation are given in Table 1.

The applied loading for this investigation was limited to the effect of internal tank pressure only. The effects of mechanical loading due to factors such as the weight of components stacked above the tank, bending loads due to wind loading, or head pressure from the acceleration of the propellant fluid were outside the scope of the present investigation. Although mechanical loading is important to the overall design of a propellant tank, the present study was primarily concerned with determining the buckling behavior of different bulkhead configurations with simple boundary effects and loading. Similarly, thermal loading was not explicitly included in the analyses; however, changes in material stiffness due to the cryogenic fluid were accounted for by using material properties that were determined at $-423^{\circ} \mathrm{F}$.

\section{Description of Bulkhead Modeling and Analyses}

Two different types of analysis codes were used to perform the analyses of the inverted bulkheads evaluated during the present investigation. The first set of analyses was conducted using the BOSOR $4^{23}$ analysis code that was developed for analyzing axisymmetric shell structures using a finite difference solution algorithm. BOSOR4 is capable of stress, stability, and vibration analyses of segmented, ringstiffened, branched shells of revolution and prismatic shells. For this investigation, two options within the BOSOR4 code were used to evaluate the bulkhead models: the "quasi-linear" analysis option and the nonlinear analysis option. The "quasi-linear" option uses nonlinear theory for the prebuckling analysis and calculates the bifurcation buckling load for a range of circumferential wave numbers using a fixed load condition. The nonlinear option uses the same nonlinear theory for the prebuckling analysis, but calculates the stability determinant for a given circumferential wave number as the load is incremented. Once a change in sign for the stability determinant is obtained, the critical buckling load corresponding to that wave number is obtained using the nonlinear prebuckling analysis.

The second set of analyses was conducted using the STAGS ${ }^{24}$ (Structural Analysis of General Shells) nonlinear shell analysis program. STAGS is a finite element code designed for the static and dynamic analysis of general shells. In addition, STAGS can perform an eigenvalue analysis for buckling and vibration based upon a linear or nonlinear stress state. The program uses both the modified and full Newton methods for its nonlinear solution algorithms, and accounts for large rotations in a shell wall by using a co-rotation algorithm at the element level. In a nonlinear analysis, STAGS performs an initial linear solution and then load or arc-length increments are automatically adjusted based upon the nonlinear response. The load and arc-length path-parameter strategy, also known as the Riks pseudo arc-length path-following method is used to continue past the limit points of a nonlinear response. In this strategy, the incrementally applied loading parameter is replaced by an arc-length along the solution path, which is then used as the independent loading parameter.

A survey of the features for each code revealed the benefit of BOSOR 4 as a relatively quick and efficient tool for conducting trade studies of axisymmetric shell structures while the STAGS code offered capabilities for performing and displaying detailed investigations into the buckling behavior of all shell structures. The benefits of using the BOSOR4 code were fast and 
efficient techniques for modeling the structure, analysis methods that included nonlinear analysis routines, and analysis routines that required very little computer time to provide a solution. In every case, the actual computer run-time was never longer than four hours using a single processor SunSparc Ultra 30 machine. This important feature of the BOSOR4 code that provided short-duration solution times for the models analyzed is due to the elimination of the circumferential dependence in the shell equations that reduced the equations from partial differential equations to onedimensional, ordinary differential equations. One drawback of the axisymmetric problem definition was that data plots generated using BOSOR4 were curvilinear representations of the shell cross-section, which lacked the ability to produce color banded, contour plots. In contrast, STAGS models were considerably more complex to generate, offered similar nonlinear solution routines, and required a great deal more computer time to obtain a solution. The advantage of using STAGS was a greater variety of solution routines, ability to model nonsymmetrical shell loading and behavior, and superior visual representations of output data.

The objectives for conducting the BOSOR4 analyses were to perform a preliminary assessment of several bulkhead configurations that would potentially affect the buckling response of the bulkhead structure and determine a single bulkhead configuration for further study. Three bulkhead shapes and thicknesses, and the effect of three sizes of supporting rings at the bulkhead to cylinder interface were evaluated to determine acceptable performance under the internal pressure loading. The three bulkhead shapes chosen for the study were a hemispherical bulkhead and two hemiellipsoidal bulkheads with major to minor (a:b) axis ratios given in Table 2. The three shell thicknesses chosen for evaluation along with three sizes of support rings are also shown. Furthermore, the thickness of each bulkhead was constant along the entire meridian in all cases. Once all the cases were evaluated, the selection of a single bulkhead configuration was made as a function of several programmatic constraints. Although all the parameters used to select the optimum configuration for an RLV derivative are unavailable, the bulkhead thickness, ring size, and dome geometry were primarily chosen based on a combination of minimum tank weight and length while maintaining an adequate margin of safety.

A description of the inverted tank geometry that was modeled using BOSOR4 and the corresponding model are shown in Figure 2. This model was used to perform a design study using the bulkhead parameters given in Table 2. Twenty-seven cases were evaluated with this model using BOSOR4, which corresponds to a full-factorial $\left(3^{3}\right)$ design. Furthermore, linear and nonlinear BOSOR4 analyses of this model were conducted using the optimum bulkhead configuration that was chosen from the design study.

The cylindrical section of the tank and the bulkhead were each modeled using one BOSOR4 shell segment that consisted of 97 mesh points. The vector normal to the curvilinear shell segments denoted by $\hat{n}$ displays the direction of the shell thickness. The length of the cylindrical shell, segment two, varied in length depending on the bulkhead shape, but was a constant fifteen inches beyond the apex of the bulkhead for each model. The thickness of segment two was a constant 0.0676 inches for the entire length except for the last six inches where the cylinder intersects the bulkhead at node 97 . At that location, a 4-inch-long built-up region with a 0.125 -inch thickness is modeled at the edge of the cylinder with a 2-inch-long transition between the two cylinder thicknesses. As previously stated, the bulkhead maintained a constant thickness along the entire meridian. Additionally, the geometry of the support ring was not explicitly modeled using shell segments, but was accounted for by using a discrete ring option within BOSOR4. Using the discrete ring option, the ring stiffness and torsional rigidity for each ring case was included in the model at node 97. The boundary conditions for the bulkhead segment, segment one, were a symmetry condition at the center of the bulkhead, node one, and free along the edge of the bulkhead. The cylindrical shell, segment two, was tied to the bulkhead at node 97 using the discrete ring and a cylinder symmetry condition at node one which was employed to hold the axial displacement and meridional rotation to zero while leaving the circumferential and radial displacements free.

The STAGS analyses were performed using two full bulkhead models that were created in MSC/PATRAN. The first model did not include a cylindrical tank section and was referred to as the bulkhead-only model while the second model included a cylindrical tank section similar to the BOSOR4 models and was referred to as the tank-bulkhead model. The bulkhead configuration that was used for all the STAGS analyses was determined from the BOSOR4 design study that will be discussed in detail later. The bulkhead-only model and the tank-bulkhead model both used the $2: 1$ hemiellipsoidal bulkhead configuration and did not include a support ring.

The bulkhead-only model had 3120 elements and approximately 18,886 degrees of freedom. The refinement of the finite element mesh of the bulkhead was limited by the aspect ratios of the triangular elements located in the center of the bulkhead. The 
boundary conditions consisted of the edge region of the bulkhead defined in a local cylindrical coordinate system in which the nodal degrees of freedom were clamped in the circumferential and axial direction similar to the BOSOR4 model conditions. The purpose of the tank-bulkhead model was to investigate the influence of the cylindrical tank boundary onto the response of the inverted bulkhead. The tank-bulkhead model consisted of the inverted bulkhead connected to a cylindrical tank wall segment. The inverted bulkhead and cylindrical tank wall had the same material properties. However, the cylindrical tank wall had a thickness of .50-inches near the outer edge of the inverted bulkhead and then tapered to a nominal wall thickness of .30 -inches for a length of 18 -inches. The tank model consisted of 5520 elements and 33,286 degrees of freedom. The boundary conditions for both models remained the same for both the linear and nonlinear STAGS buckling analyses that were conducted. In addition, an eigenvalue analysis was conducted at several nonlinear load levels for each model.

\section{$\underline{\text { Results and Discussion }}$}

A summary of the BOSOR4 results for selected cases using the "quasi-linear" option is given in Figure 3 . These results only represent a small subset of all the results that were determined during the design study, but are deemed sufficient to illustrate the behavioral trends. The operating pressure normalized with respect to the critical pressure for bifurcation buckling is plotted here as a function of the bulkhead thickness for various ratios of the bulkhead major to minor axis. Although the parameters for these cases were representative of bulkheads with variable geometry and thickness, a fixed value of $3.0 \mathrm{in}^{2}$ for the discrete ring was used. The reason for choosing a discrete ring area of $3.0 \mathrm{in}^{2}$ was two-fold. First, the effect of the discrete ring on the critical pressure for bifurcation buckling was minimal for two of the three bulkhead geometries. Also, solutions for the bulkhead with the shallowest geometry, i.e. the $2: 1$ (a:b) bulkhead, experienced convergence problems for the majority of cases due to the instability of the bulkhead. Two cases that converged to a solution had a discrete ring size of 3.0 in. $^{2}$ and were therefore chosen for presentation here.

The mode shape corresponding to the critical bifurcation buckling load using nonlinear prebuckling strains is given for the hemispherical, 1.414:1 hemiellipsoidal, and 2:1 hemiellipsoidal bulkhead geometries in Figures 4,5, and 6, respectively. In addition, each bulkhead displayed in Figures 4-6 had a discrete ring area of $3.0 \mathrm{in}^{2}$ and was 0.175 -inches thick.
After a short inspection of all three figures, it is evident that a significant change in the buckling response occurred between the hemispherical bulkhead and the shallower hemiellipsoidal bulkheads. The buckled mode shape shown in Figure 4 for the hemispherical bulkhead has a single, short half-wave along the meridian that appears to occur very close to the edge of the bulkhead with no attenuation, and corresponds to a circumferential wave number of twenty. In contrast, the mode shapes for the two hemiellipsoidal bulkheads in Figures 5 and 6 have three half-waves along the meridian that appear to occur closest to the center of the bulkhead and slowly attenuate towards the edge. Also, the number of circumferential waves that correspond to the mode shapes for the $1.414: 1$ and 2:1 hemiellipsoidal bulkheads are one and zero, respectively. These results for the hemiellipsoidal and hemispherical bulkheads correspond to the same bifurcation buckling mode shapes detailed in the investigation by Ross, et al.

Examining the data from the "quasi-linear" BOSOR4 results in Figure 3 shows a nonlinear relationship between decreasing bulkhead thickness and a corresponding decrease in the critical buckling pressure for each individual bulkhead geometry. This nonlinear relationship is evident for both the hemispherical (1:1) bulkhead geometry and the 1.414:1 hemiellipsoidal bulkhead geometry as the slope of the piecewise linear curves increase from the 0.25 -inch thickness to the 0.125 -inch thickness. In addition, the $2: 1$ bulkhead incurred a $56 \%$ decrease in the critical bifurcation buckling pressure for a corresponding 30\% reduction in the bulkhead thickness. Also, a similar nonlinear relationship appears to exist between changes in bulkhead geometry and a corresponding change in the critical buckling pressure at a constant thickness. That is, as the bulkhead becomes shallower the critical buckling load decreases more rapidly. For example, a $29 \%$ reduction in height exists from a $1.414: 1$ bulkhead to a $2: 1$ bulkhead; however, at the 0.175 -inch thickness the critical buckling pressure decreased by $50 \%$ for the same configuration.

The effect of the discrete ring on the ratios of critical buckling pressure was moderate for the hemispherical bulkhead, and very small for the hemiellipsoidal bulkheads. In the case of the hemispherical bulkhead with a bulkhead thickness of 0.125-inch thickness, the critical buckling pressure decreased by less than $1 \%$ between the 3.0 in. $^{2}$ and 1.0 in. $^{2}$ discrete ring cases; however, a $22 \%$ decrease occurred from the case with a discrete ring of $1.0 \mathrm{in}^{2}$ to the case with no ring. The effect the support ring displayed on the critical buckling pressures for the hemiellipsoidal bulkheads was almost negligible; however, as mentioned earlier in the cases for the $2: 1$ 
bulkhead geometry, the $3.0 \mathrm{in}^{2}$ ring provided the necessary stiffness to the model to allow BOSOR4 to identify the critical buckling pressure. Further evidence of the different effects the support ring had on the buckling behavior for each bulkhead can be found in their characteristic buckling responses. As noted earlier, the hemispherical bulkhead had a buckled mode shape that was predominantly confined to the edge of the bulkhead. Therefore, that bulkhead geometry appeared to be much more sensitive to changes in stiffness in the region near the bulkhead edge. Whereas, the hemiellipsoidal bulkheads had buckled mode shapes that appeared to occur mainly near the center of the bulkhead which made them relatively insensitive to changes in stiffness at the bulkhead edge.

A choice of bulkhead shape, thickness, and ring size was selected based on the "quasi-linear" results for further evaluation using BOSOR4 and STAGS. As described earlier, the configuration was primarily chosen based on the desire to determine a minimum height and weight efficient tank that met the applicable margins of safety and would provide an optimum vehicle design. This resulted in the choice of a $2: 1$ hemiellipsoidal bulkhead shape with a 0.21-inch thickness and no support ring as the optimum configuration. Using BOSOR4, additional analyses of this configuration were performed using both the "quasi-linear" option and the nonlinear analysis option. The eigenvalue corresponding to the "quasi-linear" bifurcation buckling analysis was 1.825 and the critical load factor for the nonlinear analysis of the chosen configuration was 1.602 . The critical load factor from the nonlinear analysis was obtained just prior to the BOSOR4 code indicating axisymmetric collapse of the bulkhead that was similar to that found by Corona. Thus, the convergence problems identified in some of the earlier cases using the $2: 1$ bulkhead were due to the selection of bulkhead thicknesses that were incapable of supporting the applied pressure load except for in a post-buckled configuration.

The linear buckling analysis from a linear prestress solution using STAGS was determined for the bulkhead-only model by running an eigenvalue analysis at the operating pressure of 216 psi. A critical buckling pressure of 403 psi. was determined for the bulkhead only model, which corresponds to a critical load factor of 1.864 times the 216 psi. operating pressure. An excellent agreement between the "quasi-linear" BOSOR4 results that were discussed earlier, but not presented, and the linear STAGS results was shown with approximately $2 \%$ difference between the two solutions. The corresponding mode shape, as shown in Figure 7, consisted of one axisymmetric wave that was localized in the center of the bulkhead and a much smaller wave pattern some distance outward from the first centralized wave along a meridian. Also, the mode shape obtained using STAGS closely resembled the bifurcation buckling mode shape from BOSOR4 that was given in Figure 6 for the bulkhead with a shell thickness of 0.175 inch and the same bulkhead geometry. The nonlinear solution for the bulkhead-only case required an initial analysis and three additional restart runs. The nonlinear analysis converged to a critical load factor of 1.742 or critical buckling pressure of 377 psi. A very good agreement between the nonlinear BOSOR4 results for the optimum configuration and the STAGS analyses was also shown with approximately $8 \%$ difference between the two solutions. The nonlinear response, as shown in Figure 8 , was almost identical to the linear solution except the second wave appeared to have slightly larger amplitude.

The results of the tank-bulkhead model are shown in Figure 9 as the linear buckling analysis produced a critical buckling pressure of 399 psi for this model. These results indicate that for the linear analysis the influence of the boundary edge conditions appeared negligible. However, the nonlinear analysis converged to a critical load factor of 1.631 or critical buckling pressure of 353 psi. Figure 10 shows the nonlinear response of the inverted tank-bulkhead model. The nonlinear critical buckling pressure of the tankbulkhead model was approximately $13 \%$ lower than the linear solution. These results indicate that the boundary constraint of the tank wall had some influence in lowering the critical pressure of the bulkhead.

\section{Concluding Remarks}

The BOSOR4 analysis code is an efficient sizing tool that is useful during the preliminary design phase of a practical shell structure such as an inverted bulkhead for a propellant tank. Comparison of the results between BOSOR4 and STAGS revealed a very good correlation between the two analysis methods. By selecting three important bulkhead parameters, a reasonable amount of insight into the buckling response of the shell was obtained. Although the list of parameters and range of values are not comprehensive in this investigation, the amount of computer time that would be necessary to obtain the results for a comprehensive preliminary design study for a vehicle would not be unreasonable.

An inverted bulkhead geometry is an important feature of a comprehensive tank optimization strategy that can provide the design engineer with an additional configuration for minimizing vehicle weight. The results presented here have confirmed previous work in this area by Corona and Ross, et al. while providing 
additional information on a variety of configurations and boundary effects for hemiellipsoidal bulkheads. However, as mentioned earlier, the effects of geometric imperfections on the behavior of the structures in this study were not quantified and may have a substantial impact on the results. Overall, a sufficient level of correlation has been demonstrated between the current analysis tools to provide the design engineer with enough confidence to use bulkheads that are stability critical.

The buckling characterization of an inverted, oblate bulkhead requires careful attention as small changes in bulkhead parameters can have a large effect on the critical buckling load. For example, the bulkhead with major to minor axis ratio of 2.0 incurred a $56 \%$ decrease in the critical bifurcation buckling pressure for a corresponding $30 \%$ reduction in the bulkhead thickness. Finally, since only one bulkhead configuration was examined closely, further characterization is warranted. Areas of concern that may provide significant insight are the effect of laminate construction other than quasi-isotropic, and the effect of using a tailored thickness or stiffness profile.

\section{Acknowledgements}

We would like to express our appreciation and gratitude to Dave Bushnell of LMSC for his guidance and suggestions for modeling and executing solution techniques using BOSOR4. We would also like to thank Richard Young and Mark Hilburger of NASA Langley Research Center for their valuable technical discussions that provided additional insight into the buckling of shell structures.

\section{$\underline{\text { References }}$}

1. Freeman, D.C., Wilhite, A.W., and Taley, T.A., "Advanced Manned Launch System Study Status," IAF Paper No. 91-193, $42^{\text {nd }}$ Congress of the International Astronautical Federation, Montreal, Canada, 1991.

2. Wurster, K.E., Rowell, L.F., and Peach, L.L., "The Next Generation Manned Launch System - A Complex Decision," AIAA Paper No. 93-4160, AIAA Space Programs and Technologies Conference, Huntsville, AL, September 21-23, 1993.

3. Eldred, C.H., Powell, R.W., and Stanley, D.O., "Single Stage Rocket Options for Future Launch Vehicles," AIAA Paper No. 93-4162, AIAA Space
Programs and Technologies Conference, Huntsville, AL, September 21-23, 1993.

4. Braun, R.D., Powell, R.W., Lepsch, R.A., and Stanley, D.O., "Multidisciplinary Optimization Strategies for Launch Vehicle Design," AIAA Paper No. 94-4341, $5^{\text {th }}$

AIAA/NASA/USAF/ISSMO Symposium on Multidisciplinary Analysis and Optimization, Panama City Beach, FL, September 7-9, 1994.

5. Engelund, W.C., Stanley, D.O., McMillin, M.L., and Unal, R., "Aerodynamic Configuration Design Using Response Surface Methodology Analysis," AIAA Paper No. 93-3967, AIAA Aircraft Design, Systems, and Operations Meeting, Monterey, CA, August 11-13, 1993.

6. Finckenor, J. and Bevill, M., "CORSSTOL: Cylinder Optimization of Rings, Skin, and Stringers with Tolerance Sensitivity," NASA TP3551, May 1995.

7. Sisk, D.B., "Low Profile Bulkheads for Launch Vehicle Propellant Tanks - An Optimum System Solution," AIAA Paper No. 93-4223, AIAA Space Programs and Technologies Conference and Exhibit, Huntsville, AL, September 21-23, 1993.

8. Wu, K. C. and Lepsch, R. A., "Nontangent, Developed Contour Bulkheads for a Wing-Body Single Stage Launch Vehicle," AIAA Paper No. $99-0835,37^{\text {th }}$ AIAA Aerospace Sciences Meeting and Exhibit, Reno, NV, January 11-14, 1999.

9. Corona E., "Dome Reversal of Metal Beverage Containers," J. of Pressure Vessel Tech., 120-4, November 1998, pp. 456-461.

10. Budiansky, B., "Buckling of Clamped Shallow Symmetrical Shells," Proc. of IUTAM symposium on the Theory of Thin Elastic Shells, Delft, The Netherlands, 1959, pp. 64-94.

11. Hutchinson, J.W., "Imperfections Sensitivity of Externally Pressurized Spherical Shells," J. of Applied Mechanics, 89, 1967, pp. 49-55.

12. Kaplan, A. and Fung, Y.C., "A Nonlinear Theory of Bending and Buckling of Thin Elastic Shallow Spherical Shells," NACA TN-3212, 1954.

13. Homewood, R.C., Brine, A.C., and Johnson, A.E., "Experimental Investigation of the Buckling Instability of Monocoque Shells," Proc. of the 
Society for Experimental Stress Analysis, 18, 1961, pp. 88-96.

14. Jones, E.O., "The Effects of External Pressure on Thin-Shell Pressure Vessel Heads," ASME J. of Engineering for Industry, 84, 1962, pp. 205-219.

15. Huang, N.C., "Unsymmetrical Buckling of Thin Shallow Spherical Shells," J. of Applied Mechanics, 31, 1964, pp. 447-457.

16. Galletly, G.D., Blachut, J., and Kruzelecki, J., "Plastic Buckling of Imperfect Hemispherical Shells Subjected to External Pressure," Proc. of the Institution of Mechanical Engineers, 201-C3, 1987, pp. 153-170.

17. Blachut, J., Galletly, G.D., and Moreton, D.N., "Buckling of Near-Perfect Steel Torispherical and Hemispherical Shells Subjected to External Pressure," AIAA J., 28, 1990, pp. 1971-1975.

18. Blachut, J. and Galletly, G.D., "Clamped Torispherical Shells Under External Pressure Some New Results," J. Strain Analysis, 23, 1988, pp. 9-24.

19. Blachut, J, "Buckling of Sharp Knuckle Torispheres Under External Pressure," Thin-Walled Structures, 30, 1998, pp. 55-77.
20. Lu, Z., "Imperfection Sensitivity of Elastic and Elastic-Plastic Torispherical Pressure Vessel Heads," Thin-Walled Structures, 23, 1995, pp. 2139.

21. Ross, C.T.F. and Johns, T., "Dynamic Buckling of Thin-Walled Domes Under External Water Pressure," Res Mechanica, 28, 1989, pp. 113-137.

22. Hilburger, M. H. and Starnes, J. H., Jr., "Highfidelity Analysis of Compression-loaded Composite Shells," AIAA Paper No. 2001-1394, Proceedings of the $42^{\text {nd }}$ AIAA/ASME/ASCE/AHS/ASC Structures, Structural Dynamics, and Materials Conference, Seattle, WA, 2001.

23. Bushnell, D., "Stress, Stability, and Vibration of Complex Branched Shells of Revolution Analysis and User's Manual for BOSOR4," NASA CR-2116, October 1972.

24. Rankin, C.C., Brogan, F.A., Loden, W.A., and Cabiness, H.D., "STAGS User Manual," Lockheed Martin Missiles and Space Co., Palo Alto, CA, October 1998.

Table 1. In-plane laminate material properties for the cylinder and bulkhead shell walls at $-423^{\circ} \mathrm{F}$

\begin{tabular}{lc}
\hline \hline Young's Modulus of Elasticity $\left(\mathrm{E}_{\mathrm{x}}\right.$ and $\left.\mathrm{E}_{\mathrm{y}}\right), \mathrm{Msi}$ & 8.77 \\
\hline Shear Modulus $\left(\mathrm{G}_{\mathrm{xv}}\right), \mathrm{Msi}$ & 3.76 \\
\hline Poisson's Ratio $\left(\mathrm{v}_{\mathrm{xv}}\right)$ & 0.299 \\
\hline \hline
\end{tabular}

Table 2. Minimum, intermediate, and maximum values used to define the bulkhead configurations for the BOSOR4 analyses

\begin{tabular}{ccc}
\hline \hline $\begin{array}{c}\text { Bulkhead shape } \\
(\mathrm{a} / \mathrm{b})\end{array}$ & $\begin{array}{c}\text { Bulkhead thickness } \\
\text { (in.) }\end{array}$ & $\begin{array}{c}\text { Ring area } \\
\text { (in. }{ }^{2} \text { ) }\end{array}$ \\
\hline 1.00 & $0.125,0.175,0.250$ & $0.00,1.00,3.00$ \\
\hline 1.414 & $0.125,0.175,0.250$ & $0.00,1.00,3.00$ \\
\hline 2.00 & $0.125,0.175,0.250$ & $0.00,1.00,3.00$ \\
\hline \hline
\end{tabular}




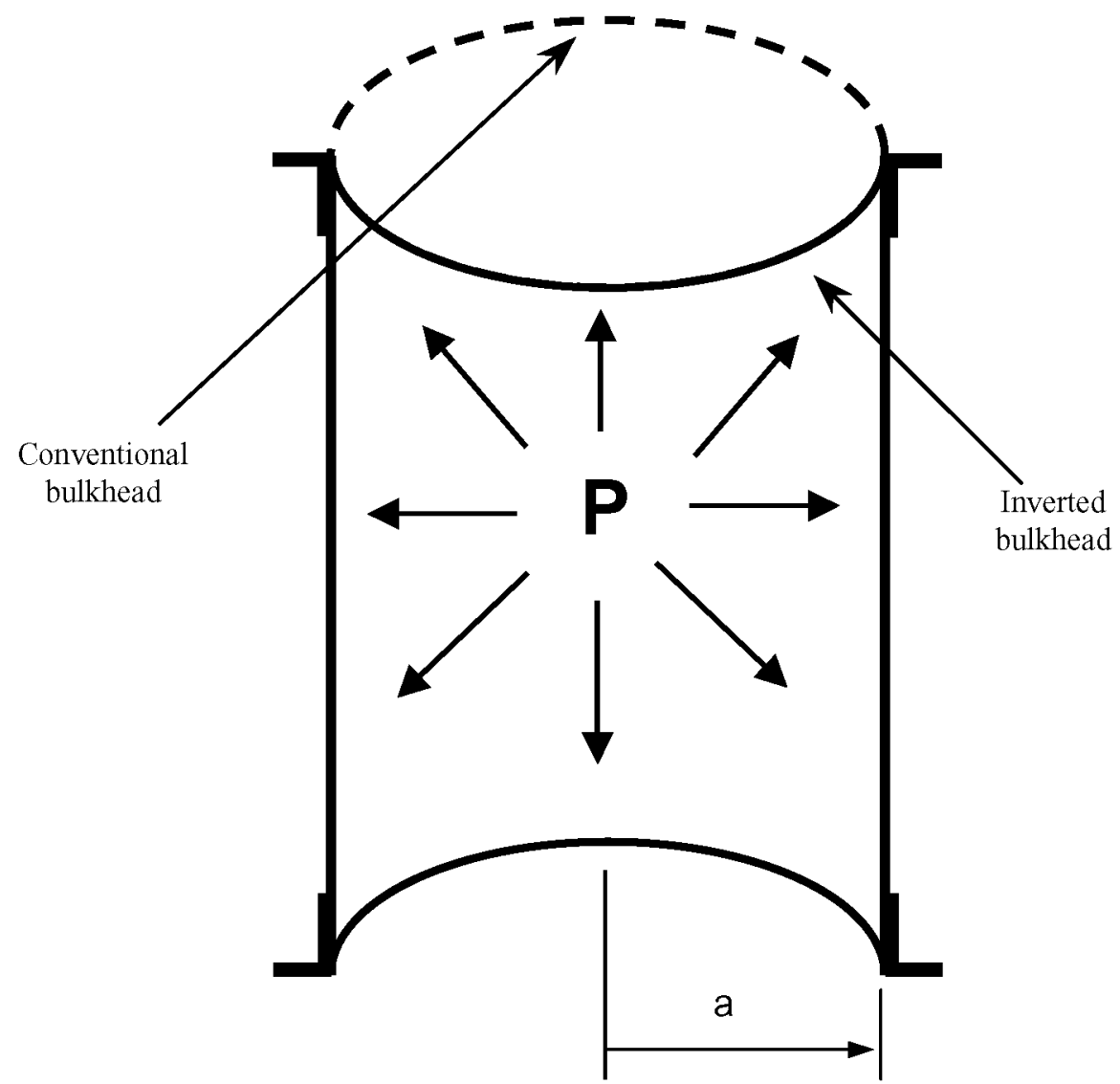

Figure 1. Cross-section of a propellant tank with conventional and inverted bulkhead geometries.

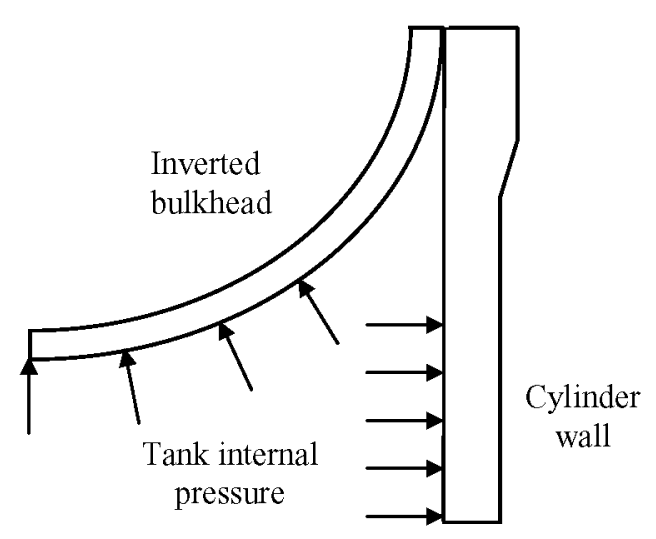

a) Description of the cylinder and inverted bulkhead geometry

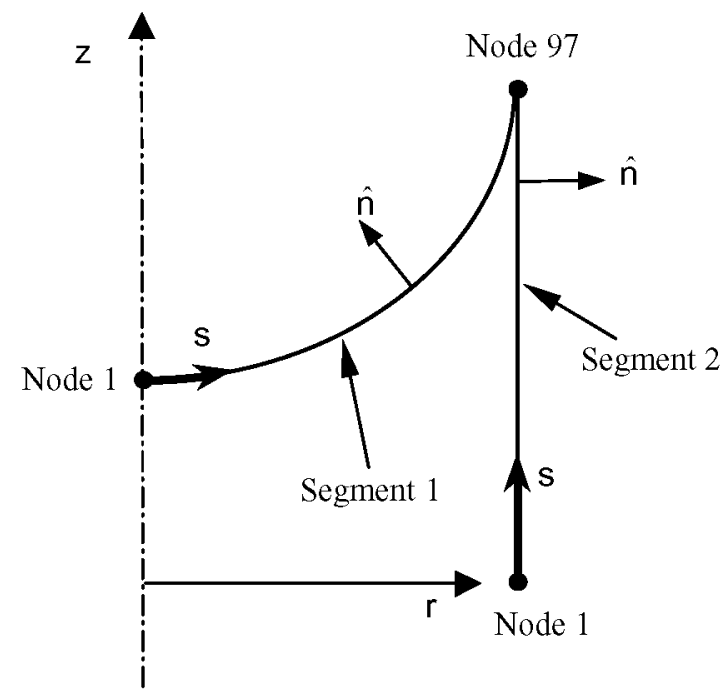

b) Model displaying nodes along the reference surfaces

Figure 2. Structural definition for BOSOR4 analyses. 


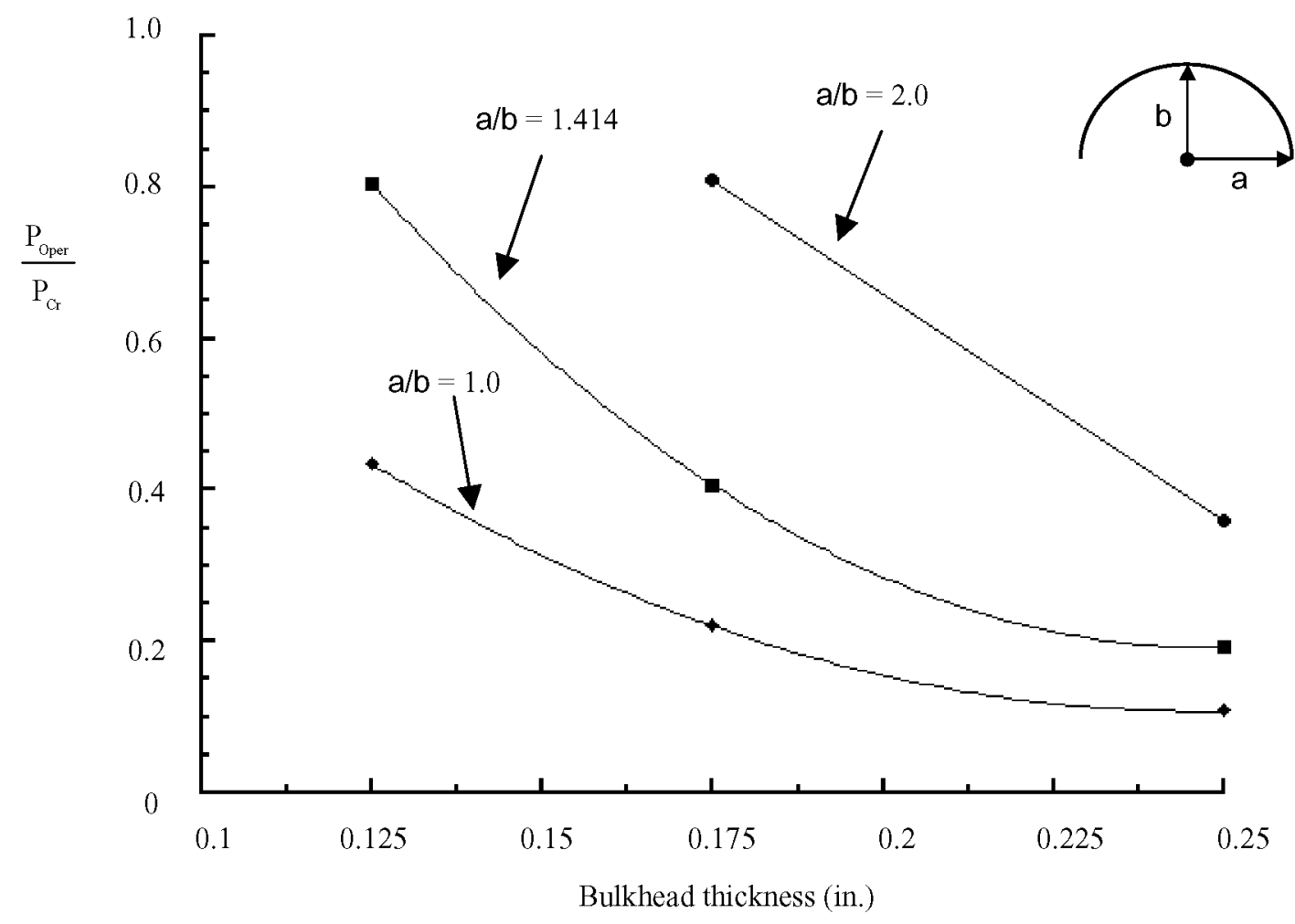

Figure 3. Ratio of operating pressure to critical buckling pressure results from the BOSOR4 models as a function of bulkhead thickness for a ring area of 3.0.

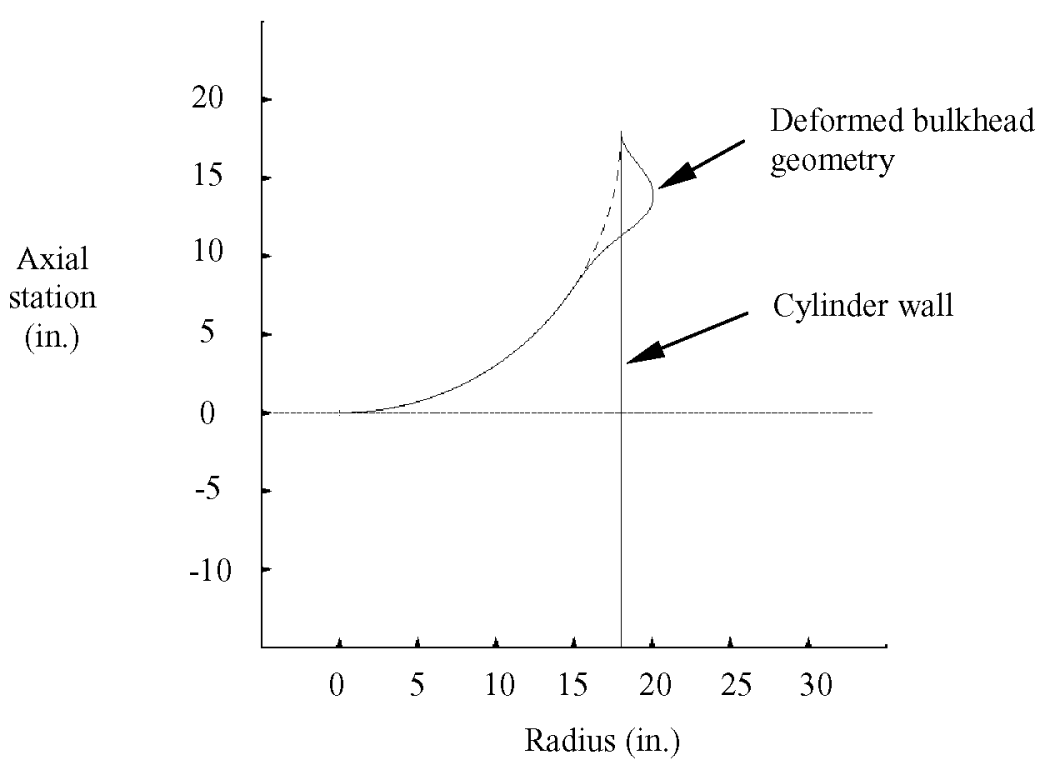

Figure 4. Bulkhead mode shape from BOSOR4 linear analysis corresponding to the critical buckling pressure of 980 psi. with a hemispherical geometry and 0.175 -inch shell thickness. 


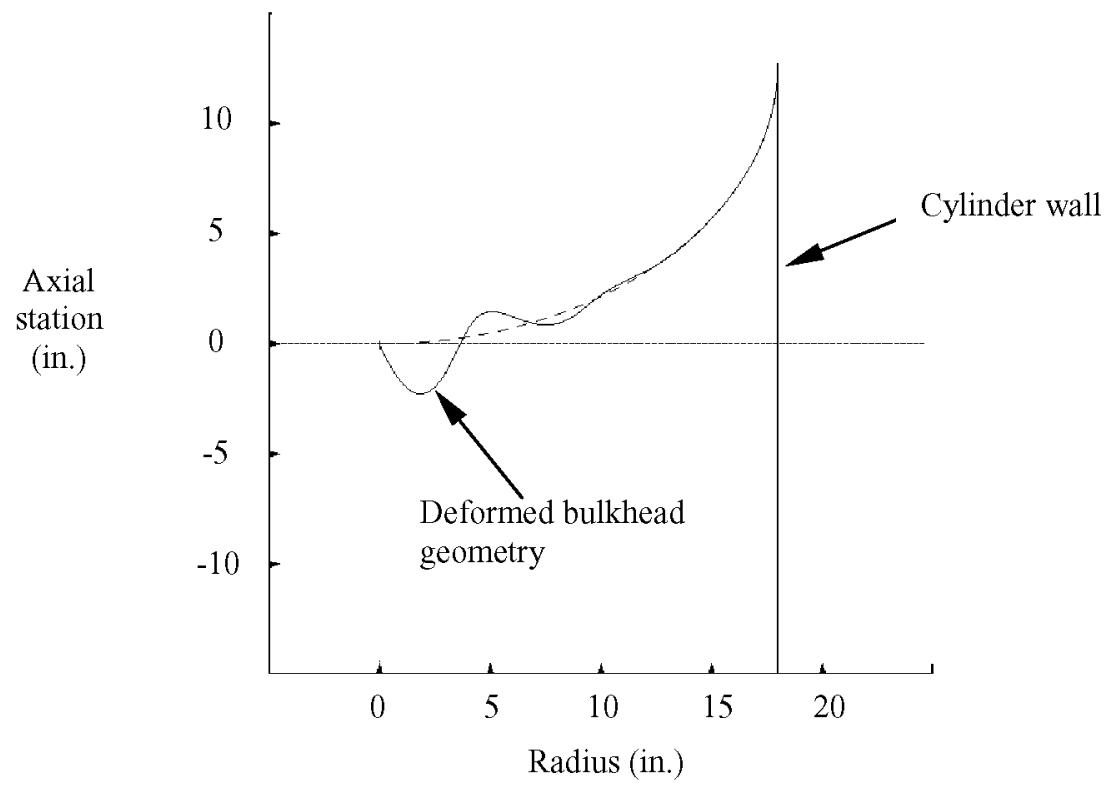

Figure 5. Bulkhead mode shape from BOSOR4 linear analysis corresponding to the critical buckling pressure of 530 psi. with a 1.414:1 hemiellipsoidal geometry and 0.175-inch shell thickness.

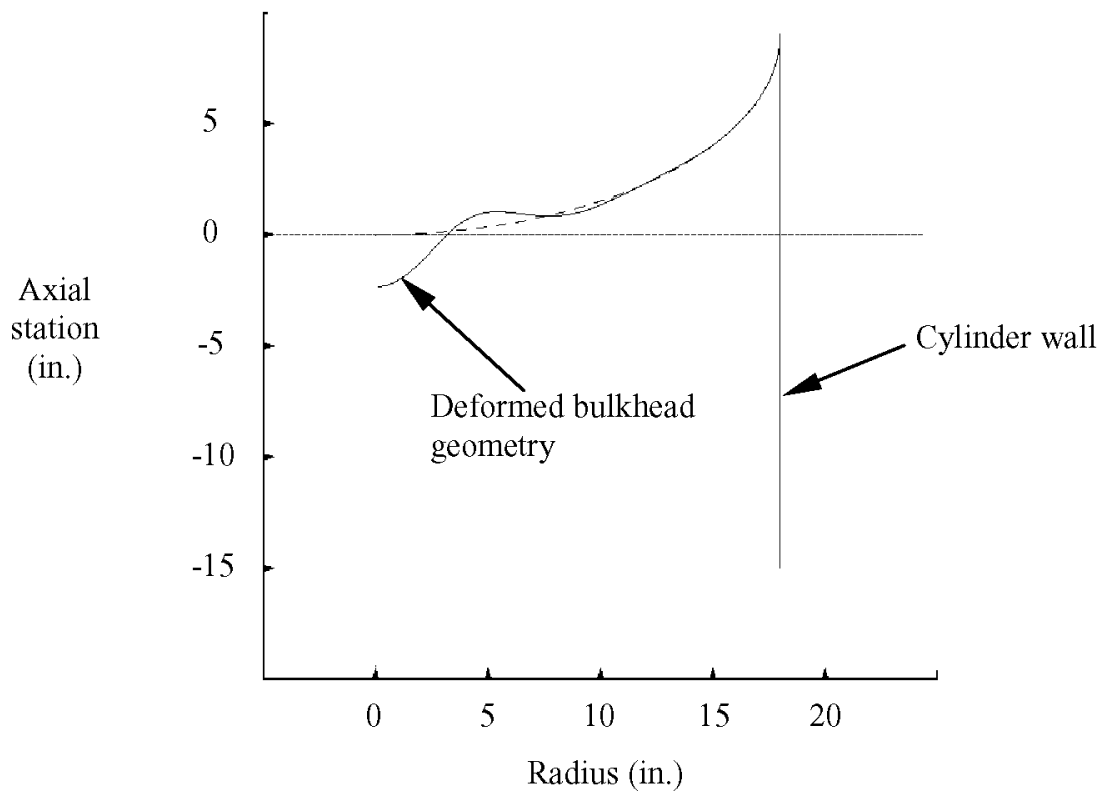

Figure 6. Bulkhead mode shape from BOSOR4 linear analysis corresponding to the critical buckling pressure of 267 psi. with a 2:1 hemiellipsoidal geometry and 0.175 -inch shell thickness. 


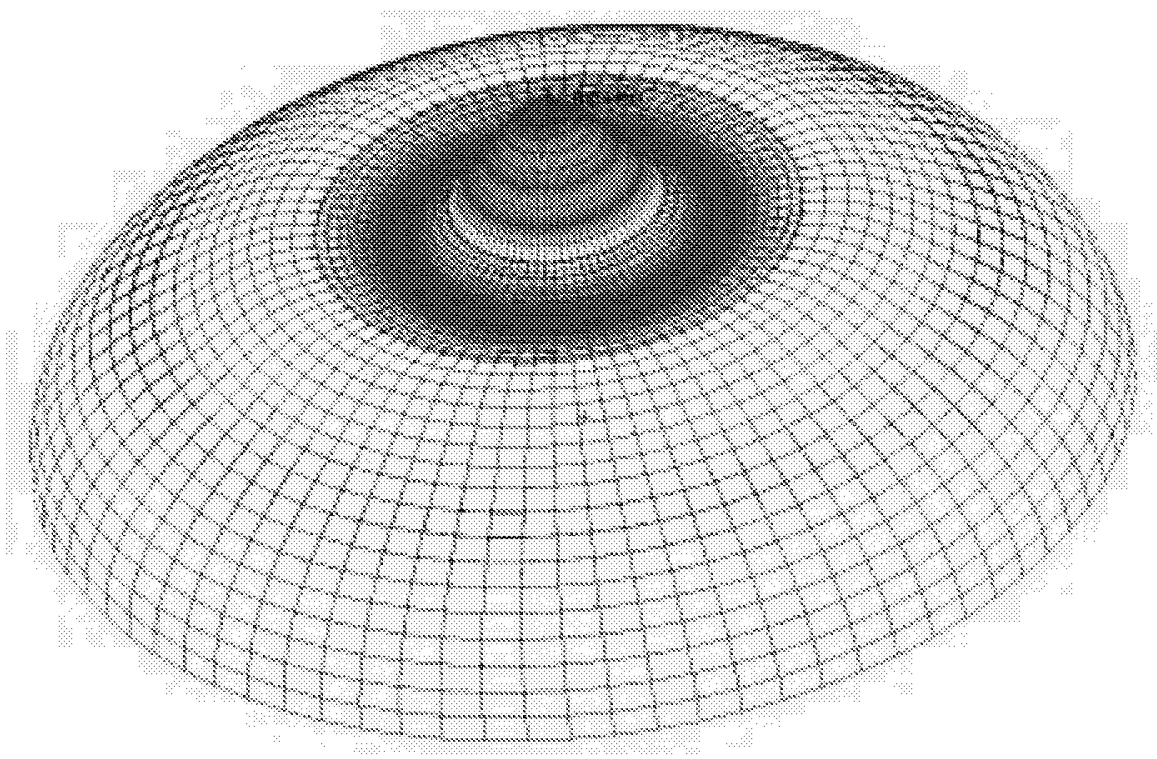

Figure 7. Bulkhead mode shape from STAGS linear analysis corresponding to the critical buckling pressure of 403 psi. for the bulkhead-only model

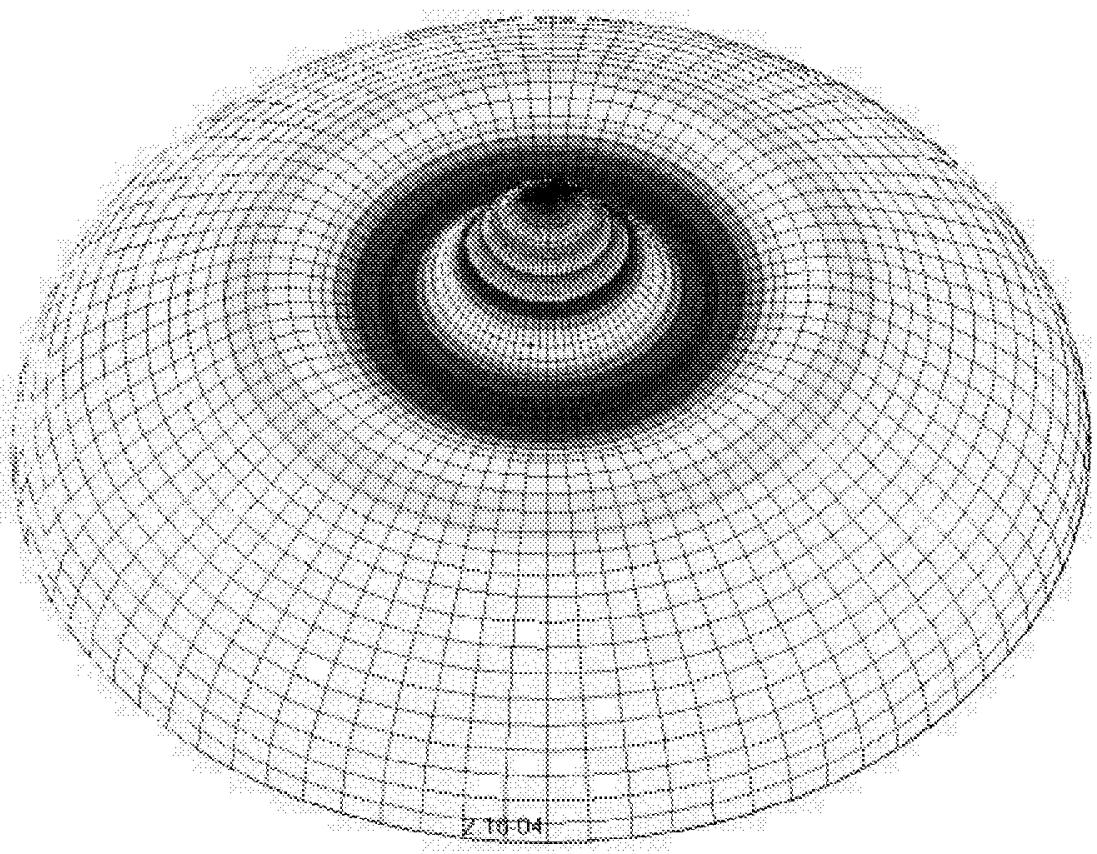

Figure 8. Bulkhead mode shape from STAGS nonlinear analysis corresponding to the critical buckling pressure of 377 psi. for the bulkhead-only model. 


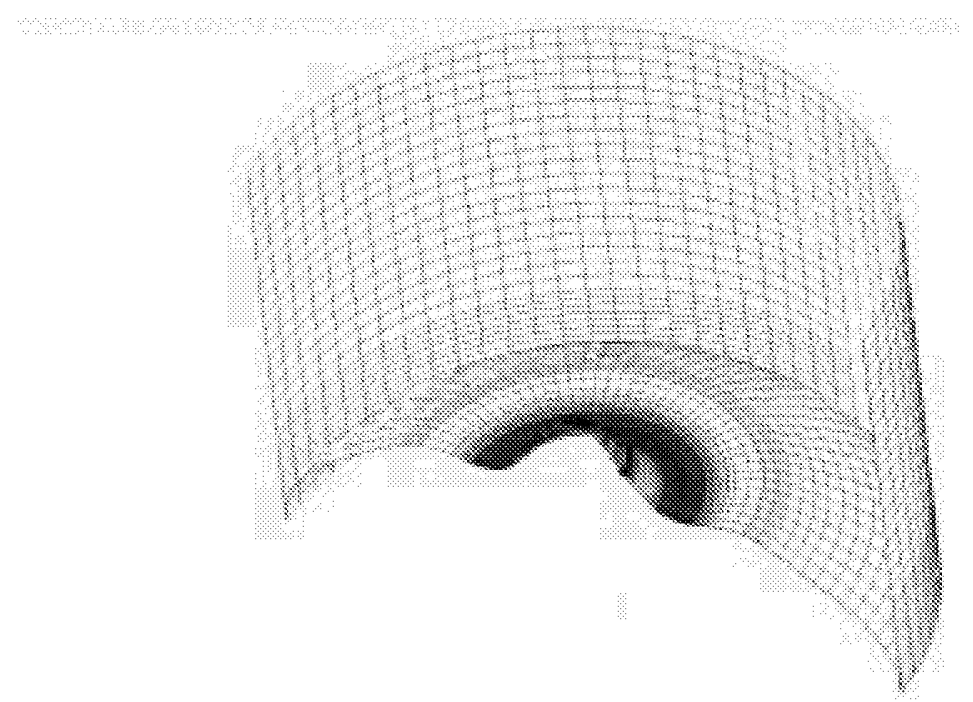

Figure 9. Cylinder and bulkhead mode shape from STAGS linear analysis corresponding to the critical buckling pressure of 399 psi. for the tank-bulkhead model.

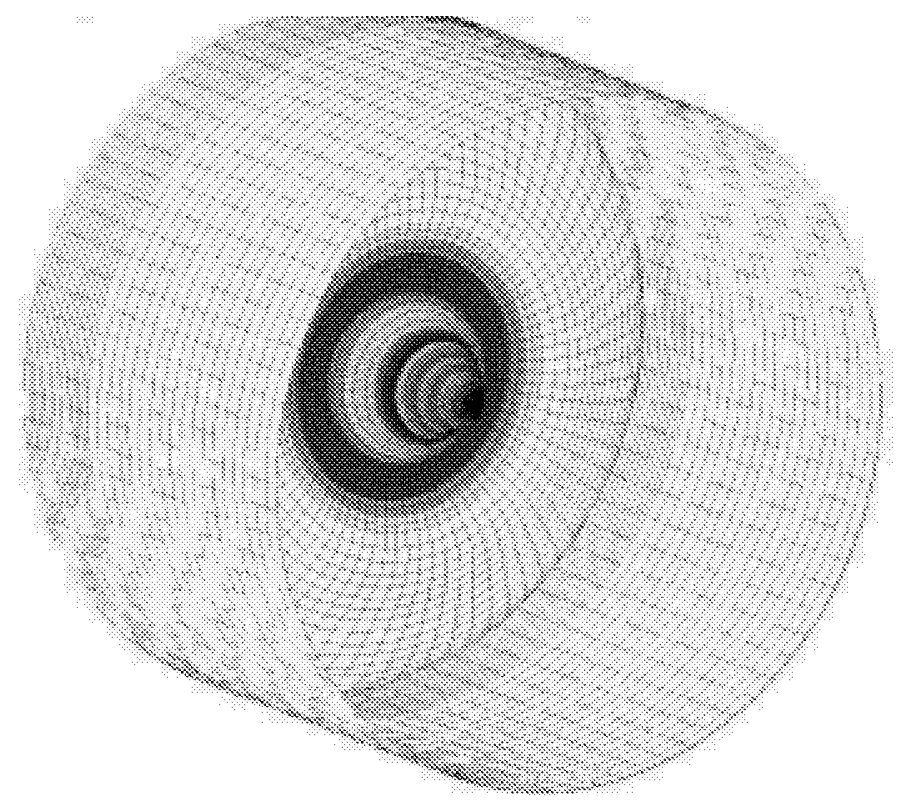

Figure 10. Cylinder and bulkhead mode shape from STAGS nonlinear analysis corresponding to the critical buckling pressure of 353 psi. for the tank-bulkhead model. 